\title{
Plasma Levels of Heat Shock Protein 90 Alpha Associated With Colorectal Cancer Development
}

\author{
Wene Wei ${ }^{1 \dagger}$, Jiahui Zhou ${ }^{1 \dagger}$, Lipeng Chen ${ }^{2}$, Haizhou Liu ${ }^{1}$, Fuyong Zhang ${ }^{3}$, Jilin Li $^{1}$, \\ Shufang Ning ${ }^{1}$, Shirong $\mathrm{Li}^{1}$, Chen Wang ${ }^{1}$, Yi Huang ${ }^{1}$, Chang Zou ${ }^{2 \star}$ and Litu Zhang ${ }^{1 *}$ \\ ${ }^{1}$ Department of Research, Guangxi Medical University Cancer Hospital, Nanning, China, ${ }^{2}$ Shenzhen People's Hospital, The \\ Second Clinical Medical College, Jinan University, The First Affiliated Hospital, Southern University of Science and Technology, \\ Shenzhen, China, ${ }^{3}$ Department of Laboratory, The First Affiliated Hospital of Guangxi Medical University, Nanning, China
}

OPEN ACCESS

Edited by:

Tianhui Chen,

University of Chinese Academy of

Sciences, China

Reviewed by:

Salva Mena-Mollá

University of Valencia, Spain

Prihantono Prihantono,

Hasanuddin University, Indonesia

Bo Wei,

Third Affiliated Hospital of Sun Yat-sen

University, China

${ }^{*}$ Correspondence:

Chang Zou

zou.chang@szhospital.com

Litu Zhang

zhanglitu@gmail.com

${ }^{t}$ These authors have contributed equally to this work

Specialty section:

This article was submitted to

Molecular Diagnostics and

Therapeutics,

a section of the journal

Frontiers in Molecular Biosciences

Received: 24 March 2021 Accepted: 25 June 2021

Published: 08 July 2021

Citation:

Wei W, Zhou J, Chen L, Liu H, Zhang F, $L i$ J, Ning S, Li S, Wang C, Huang $Y$,

Zou C and Zhang L (2021) Plasma Levels of Heat Shock Protein 90 Alpha Associated With Colorectal

Cancer Development.

Front. Mol. Biosci. 8:684836. doi: 10.3389/fmolb.2021.684836
Aim: The role of plasma heat shock protein 90 alpha (HSP90a) in colorectal cancer patients remains unclear. This study aimed to evaluate the relationship between HSP9Oa and the occurrence and development of colorectal cancer through diagnosis and prognosis value.

Methods: 635 colorectal cancer patients and 295 healthy controls were recruited. The HSP90a was measured by using the ELISA kit in all objects and the immune cells and common biomarkers as CEA, AFP, CA125, CA153 and CA199 were measured in all colorectal cancer patients. The relationship between plasma HSP90a with clinical features, common tumor markers and immune cells were also conducted. The survival analysis endpoint was progression-free survival (PFS).

Results: The levels of plasma HSP90a were significantly higher in colorectal cancer patients compared to healthy controls [51.4 (ng/ml) vs. $43.7(\mathrm{ng} / \mathrm{ml}), p<0.001]$. In additional, the levels of plasma HSP9Oa were associated with gender and disease progress as stage, lymphatic and distant metastasis. Furthermore, plasma HSP90a was closed correlation with CEA, CA125, CA199 and percentage of B cells. However, the initial expression level of plasma HSP90a failed to show a prognostic value for progression-free survival in colorectal cancer.

Conclusion: The plasma Hsp90a was remarkable higher in colorectal cancer and correlated with common tumor biomarkers and immune cells. Plasma Hsp90a levels were associated with disease progress but a poor diagnosis performance and also failed to show a prognostic value in colorectal cancer.

Keywords: heat shock protein 90 alpha, colorectal cancer, metastasis, diagnosis, prognosis

\section{INTRODUCTION}

Colorectal cancer (CRC) is one of the most common tumors worldwide. Approximately 1.8 million people were diagnosed with colorectal cancer and 861,000 died from colorectal cancer in 2018, which equates to 4,931 people diagnosed with colorectal cancer and 2,358 people dying from colorectal cancer every day (Bray et al., 2018). Metastasis and recurrence have always been difficult points in cancer treatment. The liver is the most common site of distant metastases and accounts for $20 \%$ of 
CRC patients who arrive in hospitals with synchronous disease, and approximately $50 \%$ of CRC patients develop liver metastases at some point in the course of their disease (Leonard et al., 2005; Manfredi et al., 2006). With the development of medical technology, the treatments for colorectal cancer patients include endoscopic and surgical local excision, downstaging preoperative radiotherapy and systemic therapy, extensive surgery for locoregional and metastatic disease, local ablative therapies for metastases, and palliative chemotherapy, targeted therapy, and immunotherapy (Hurwitz et al., 2004; André et al., 2009; Van Cutsem et al., 2009; Haller et al., 2011; Ahlenstiel et al., 2014; Baratti et al., 2016; Ma et al., 2017; Abdel-Rahman and Cheung, 2018). Distant metastatic CRC (mCRC) patients have historically been associated with poor survival. Without treatment, the median survival time of mCRC patients is < 12 months, and the 5 years survival rate is $<10 \%$ (Mohammad and Balaa, 2009; Morris and Treasure, 2018). Undergoing bevacizumab in combination with oxaliplatin or capecitabine chemotherapy therapy, patients with colorectal cancer have a significantly improved prognosis, with a median progression-free survival (PFS) of approximately 9 months (Saltz et al., 2008; Cunningham et al., 2013). Patients with metastatic colorectal cancer have a good survival benefit from prompt and aggressive treatment. Therefore, timely detection of metastasis is particularly important for colorectal cancer patients.

Traditionally, computed tomography (CT) scan is the common modality for initial diagnosis of distant metastasis and the modality of choice in order to discover those patients for evidence of a recurrence of the disease (Benson et al., 2013). However, preoperative chemotherapy or neo-adjuvant therapy would generally decrease the sensitivity of CT scans as a method for monitoring metastasis in CRC patients (van Kessel et al., 2012; Rojas Llimpe et al., 2014). Therefore, an optimal strategy for early detection and diagnosis distant metastasis for CRC patients is essential. Meanwhile, an appropriate way to assess prognosis is also urgently needed.

Due to the accessibility and noninvasiveness advantages, peripheral blood samples are commonly used for biomarker determinations and are widely accepted as an acceptable intervention by patients and health workers. In addition to commonly used tumor markers such as CEA, AFP, CA125, CA153 and CA199, heat shock protein 90 alpha (HSP90a) is a broad-spectrum tumor marker worthy of attention in recent years. HSP90a is an intracellular molecular chaperone which is exposed to the extracellular space. It has been documented that the overexpression of HSP90a was associated with tumor development, progression, invasiveness, metastatic potentials and chemo-resistance in various types of cancers (Passarino et al., 2003; Eustace et al., 2004; Tsutsumi et al., 2008; Chang et al., 2009; Zuehlke et al., 2015). Previous studies showed that HSP90 a levels with or without AFP can act as excellent diagnostic markers for liver cancer (Fu et al., 2017; Wei et al., 2020). In addition, HSP90 $a$ also showed a good diagnostic performance for lung cancer and early CRC patients (Shi et al., 2014; Kasanga et al., 2018). In China, HSP90a was approved for clinical application as a broad-spectrum tumor marker in 2016. However, the diagnostic and prognostic efficacies of plasma
HSP90a in patients with colorectal cancer has not been thoroughly confirmed. In the current study, we will assess the diagnostic and prognostic value of HSP90a for CRC patients.

\section{MATERIAL AND METHODS}

\section{Patients}

635 CRC patients were recruited from the Department of Gastrointestinal Surgery, Guangxi Medical University Cancer Hospital from 1, Jan, 2018 to 31, Aug, 2019 with a median age 60 years. The inclusion criteria for all patients that there was: 1) confirmation of CRC by clinical manifestation and histopathological examination associated with imaging diagnosis; 2 ) the state of distant metastases was assessed; 3) no anti-tumor treatment or surgical resection were performed at the time of diagnosis; 4) the tumor markers, immune cells and HSP90a in peripheral blood were evaluated before any treatment; 5) complete information for clinical features were available. The exclusion criteria for all patients were if they had: 1) CRC combined with other cancers; 2) a history of malignant tumors in other organs; 3 ) radiation or chemotherapy prior to admission; 4) distant metastases which could not be evaluated. The control group included 170 patients from The First Affiliated Hospital of Guangxi Medical University who had received health examinations during the same period with a median age 37.5 years. Stages for patients with colorectal cancer were classified according to the American Joint Committee on Cancer Classification (the 7th edition). Patients were followed up for tumor assessments every 12 weeks. Disease progression assessed by using RECIST 1.1 (Eisenhauer et al., 2009).

\section{Methods}

Detection of peripheral blood markers was performed as follows. Fasting elbow venous blood was collected in EDTA anticoagulant tubes to prepare plasma samples and in dried tubes to prepare serum samples. All samples were separated by centrifugation at 3,000 rpm for $10 \mathrm{~min}$. The levels of plasma HSP90a were measured by using the ELISA kit for HSP90a protein (Yantai Protgen Biotechnology Development Co., Ltd., Yantai, China). All operations followed the manufacturer's instructions. The kit was pre-incubated at $37^{\circ} \mathrm{C}$ for $30 \mathrm{~min}$ and the plasma samples were diluted 20 times with diluent solution provided in the kit. The standards were loaded together with the quality controls and the prepared samples $(50 \mu \mathrm{L}$ of each) added into 96-well plates followed by addition of $50 \mu \mathrm{L}$ of anti-Hsp90aHRP-conjugated antibody. Then, the plate was incubated at $37^{\circ} \mathrm{C}$ for $1 \mathrm{~h}$ after gentle shaking. Next, the color reaction step was carried out after six washes. $50 \mu \mathrm{L}$ of peroxide and $50 \mu \mathrm{L}$ of $3,3^{\prime}, 5,5^{\prime}$-tetramethylbenzidine solution was added and the mixture was incubated at $37^{\circ} \mathrm{C}$ for a further $20 \mathrm{~min}$ followed by termination of the reaction with an acid stop buffer. Finally, the optical density was measured using a spectrophotometer at $450 \mathrm{~nm}$ for the detection wavelength, with $620 \mathrm{~nm}$ as the reference wavelength. The concentration of HSP90a protein in each sample was calculated according to a standard curve of optical density values. Meanwhile, the expression status of HSP90a in colon adenocarcinoma (COAD) was also evaluated by using data retrieved from The Cancer Genome Atlas (TCGA). 
The levels of serum CEA, AFP, CA125, CA153 and CA19-9 were measured by chemiluminescence microparticle immuno assays (CMIA) using the Architect i2000SR analyzer and the corresponding reagent kits which were purchased from Architect Diagnostics, America. All operations followed the manufacturer's instructions. The immune cells in this study including $\mathrm{T}$ cells, helper $\mathrm{T}$ lymphocytes (Th cells), suppressor $\mathrm{T}$ lymphocytes (Ts cells), Natural killer cells (NK cells) and B cells were defined as CD45+/CD3+ lymphocytes, CD45+/CD3+/CD4+ lymphocytes, CD45+/CD3+/CD8+ lymphocytes, CD45+/CD3-/CD16+/CD56 + lymphocytes and CD45+/cCD19 + lymphocytes, respectively. Flow cytometry (BD Biosciences, Franklin Lakes, NJ, United States) was performed to detect labeled cells and analyze the results.

\section{Statistical Analysis}

Levels of the HSP90a, CEA, AFP, CA125, CA153 and CA19-9 were assessed by SPSS STATISTICS 23.0 (IBM, Chicago, IL, United States) to ascertain normal distribution and the data are presented as medians and ranges. Nonparametric KruskalWallis H-test was used to compare the differences between groups. Wilcoxon rank sum test was used to analyze HSP90a expression in COAD samples from The Cancer Genome Atlas (TCGA). Correlation between the indicators was analyzed using Pearson analysis. The diagnostic value was analyzed using receiver operating characteristic (ROC) curves, with the area under the curve (AUC). The optimal cut-off values for ROC curves were established using the Youden Index (YI = sensitivity + specificity -1). Progression-free survival (PFS) was the primary study end point, and was defined as the time from initial diagnosis to the date of disease recurrence and was censored at the last follow-up or at the time of death from any cause. The survival curves were generated using the Kaplan-Meier curve and survival differences were estimated by a log-rank test. $p<0.05$ was considered statistically significant.

\section{RESULTS}

\section{Basic Profile of Tumor Markers and Immune Cells in Patients With Colorectal Cancer}

This study recruited 635 cases of colorectal cancer patients and 295 cases of healthy controls. Routine tumor markers and immune cells were assessed in all patients and the results were showed in Table 1. The plasma levels of HSP90a protein were significantly higher in patients with colorectal cancer than healthy controls [51.4 $(33.8,80.3) \mathrm{ng} / \mathrm{ml}$ vs. $43.7(34.3,54.8) \mathrm{ng} / \mathrm{ml} ; p<$ 0.001 , Figure 1]. There was no significant difference in plasma HSP90a protein levels among healthy controls with different age distribution (all $p>0.05$, Figure 1).

\section{Correlations Between the Plasma Levels of HSP90a Protein and Clinic-Pathological Characteristics in Colorectal Cancer Patients}

The associations of plasma levels of HSP90a protein and clinic-pathological characteristics in colorectal cancer patients were showed in Table 2. Male patients with colorectal cancer were showed a significantly higher plasma level of HSP90a protein $(p=0.002)$. Meanwhile, the plasma levels of HSP90a protein were correlated with disease stage, lymphatic metastasis, and distant metastasis (all $p<0.001)$. There was no difference in plasma HSP90a protein expression among different pathological differentiation levels $(p=0.153)$.

\section{Correlations Between the Plasma Levels of HSP90a Protein With Routine Tumor Markers and Immune Cells}

The correlations between tumor markers and immune cells percentage were showed in Figure 2. There was no statistical correlation between common tumor markers CEA, AFP, CA125, CA153, CA199, TK1 and immune cells in patients with colorectal cancer (all $p>0.05$, Figure 2). Plasma HSP90a protein levels were positively related to CEA, CA125, CA199 and negatively related to B lymphocyte percentage (all $p<0.05$, Figure 2).

\section{Univariate and Multivariate Analysis for Factors Associated With Metastasis in Colorectal Cancer}

The univariate and multivariate analysis for factors associated with metastasis in colorectal cancer were showed in Table 3. The univariate analysis showed that the biomarkers as CA125, CEA, CA199 and HSP90a were associated with metastasis in colorectal cancer patients, and the multivariate analysis revealed that the biomarkers as CEA, CA199 and HSP90a were independent risk factor for distant metastasis in patients with colorectal cancer (all $p<0.001$ ). A nomogram for predicting the presence of metastasis in patients with colorectal cancer was showed in Figure 3.

TABLE 1 | Basic profile of tumor markers and immune cells in patients with colorectal cancer.

\begin{tabular}{lccc}
\hline Parameters & Colorectal cancer & Healthy control & $\boldsymbol{p}$ value \\
\hline CEA $(\mathrm{ng} / \mathrm{ml})$ & $5.3(2.4,17.8)$ & $0.9(0.6,1.4)$ & $<0.001$ \\
AFP $(\mathrm{ng} / \mathrm{ml})$ & $2.5(1.9,3.4)$ & $2.1(1.6,2.8)$ & $<0.001$ \\
CA125 $(\mathrm{U} / \mathrm{ml})$ & $11.1(7.7,18.7)$ & $11.7(8.3,17.2)$ & 0.952 \\
CA153 (U/ml) & $9.3(6.2,13.3)$ & $16.0(13.0,22.7)$ & $<0.001$ \\
CA199 (U/ml) & $9.1(3.5,41.4)$ & $12.9(7.4,17.9)$ & 0.090 \\
HSP90a $(\mathrm{ng} / \mathrm{ml})$ & $51.4(33.8,80.3)$ & $43.7(34.3,54.8)$ & $<0.001$ \\
TK1 $(\mathrm{ng} / \mathrm{ml})$ & $0.8(0.4,1.3)$ & - & - \\
T cells $(\%)$ & $66.1(58.7,72.13)$ & - & - \\
Th cells $(\%)$ & $39.7(33.9,45.5)$ & - & - \\
Ts cells $(\%)$ & $19.3(15.2,24.2)$ & - & - \\
Ratio & $2.0(1.6,2.7)$ & - & - \\
NK $(\%)$ & $13.1(8.7,18.8)$ & - & - \\
B cells $(\%)$ & $12.0(9.3,15.7)$ & & - \\
\hline
\end{tabular}

Ratio: the ratio of the Th cells to Ts cells. All values are presented in median and quartile intervals. The $\mathrm{p}$ values were shown in italics. 


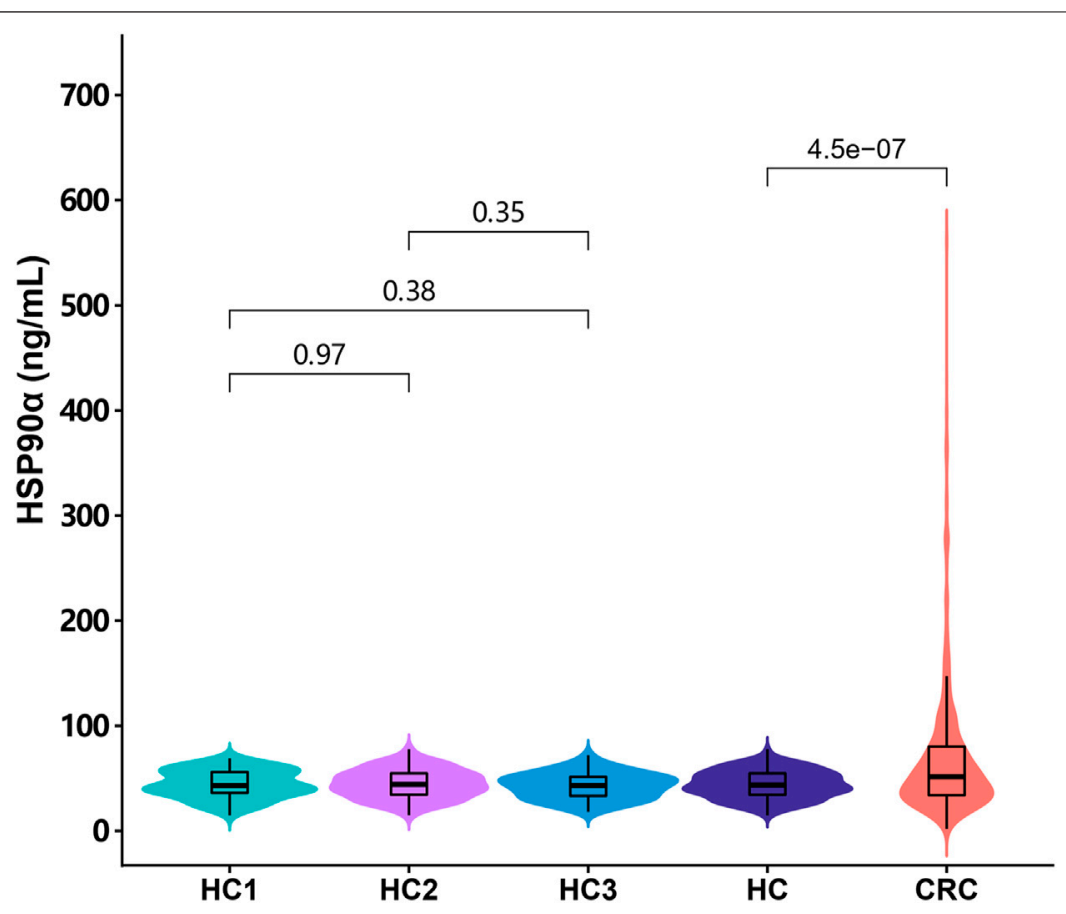

FIGURE 1 | The distribution of the plasma HSP90 a protein expression in colorectal cancer patients and healthy controls. HC1: subgroup under 40 years of age in healthy control group; HC2: subgroup of healthy controls aged 40-59 years; HC3: subgroup over 60 years of age in healthy control group; HC: all healthy controls, CRC: colorectal cancer.

TABLE 2 | Correlations of the plasma HSP90 $\alpha$ with clinicopathologic features in colorectal cancer.

Parameters

Age ( $<60$ years, $\geq 60$ years)

Gender (male, female)

Differentiation (well, moderately, poor)

Stage (I-II, III-IV)

Lymphatic metastasis (absent/present)

Metastasis (absent/present)

\section{Frequency}

$315 / 320$

$414 / 221$

$25 / 545 / 65$

$201 / 434$

$233 / 402$

$470 / 165$
HSP90a (ng/ml)

$p$ value

$51.4(33.8,80.3) / 48.2(32.5,72.6)$

$54.5(35.9,84.7) / 43.7(30.2,71.7)$

0.073

0.002

$48.1(31.0,72.3) / 50.5(33.3,78.3) / 58.0(38.5,90.8)$

$43.5(30.1,63.0) / 55.6(35.4,88.1)$

$43.3(30.7,62.5) / 57.1(35.7,92.8)$

$45.3(30.9,70.6) / 72.6(43.3,131.0)$
0.153

$<0.001$

$<0.001$

$<0.001$

All values are presented in median and quartile intervals. The results of $\mathrm{p}<0.05$ were highlighted in bold italics.

The Diagnostic Efficacy of Plasma HSP90a in Colorectal Cancer and its Ability to Distinguish Colorectal Cancer Patients With Distant Metastasis

ROC analysis was conducted to assess the diagnostic ability of plasma HSP90a for colorectal cancer and results were showed in Figure 4. Using serum CEA, CA199 and plasma HSP90a levels of healthy donors as control group, the serum CEA has significant advantages in colorectal cancer diagnosis with a cut-off value $1.77 \mathrm{ng} / \mathrm{ml}$ (AUC $=0.944$, sensitivity $86.3 \%$, specificity $87.12 \%$, Figure 3A). However, the plasma HSP90a levels showed a poor performance in colorectal cancer diagnosis with a cut-off value $69.1 \mathrm{ng} / \mathrm{ml}$ (AUC $=0.602$, sensitivity $33.1 \%$, specificity $99.0 \%$, Figure 3A). Patients with colorectal cancer were divided into M0 and $\mathrm{M} 1$ groups according to the absence or presence of distant metastasis. Using the M0 group as controls, the AUCs of CEA,
CA19-9, HSP90 $\alpha$ and the panel for distinguishing the presence of distant metastasis from CRC patients were 0.776, 0.755, 0.690, 0.819 , respectively (Figure 3B). A nomogram for predicting the presence of metastasis in patients with colorectal cancer was showed in Figure 4, higher total score based on the sum of the assigned numbers for each of the factors in the nomogram was associated with a high risk of mCRC.

\section{Correlations Between Concentrations of HSP90 $\alpha$, CEA and CA19-9 and PFS in CRC Patients}

Of 635 colorectal cancer patients, 454 cases had complete follow-up data and received standardized treatment. The median follow-up time was 7 months (range from 1 to 32 months). Survival analysis was conducted based on the median of CEA, CA19-9 and HSP90 a levels in all colorectal cancer patients and the cutoff values calculated according 


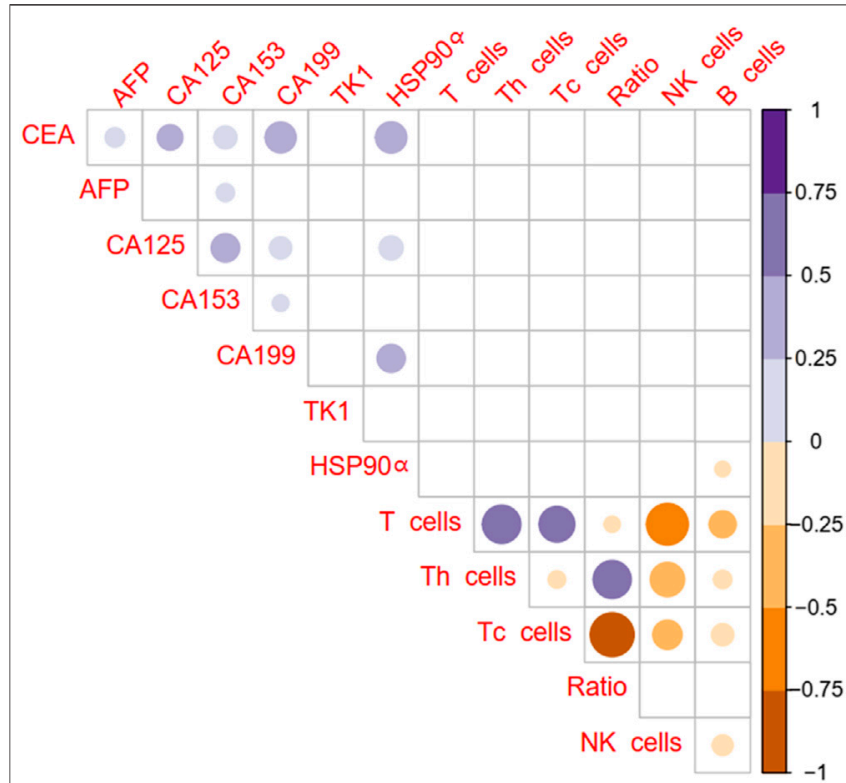

FIGURE 2 | Correlation of plasma HSP90a protein levels with routine tumor markers and immune cells in colorectal cancer patients (Pearson analysis). Blue color represents a positive correlation, brown color represents a negative correlation, and the shade of color represents the degree of correlation. The results with $p<0.05$ were filled with color and display in the diagram.

to Yuden index respectively, and the results are shown in Figure 5. The initial concentrations of CEA, CA19-9 and HSP90a were not correlated with PFS in colorectal cancer (all $p>0.05$, Figure 5).

\section{HSP90a Expression in TCGA Colorectal Cancer}

To further explore the expression status of HSP90a in colon adenocarcinoma (COAD), we analyzed the expression data of HSP90 $\alpha$ in COAD samples using data retrieved from The
Cancer Genome Atlas (TCGA), and found that the expression levels of HSP90a in tumor samples were significantly higher than these from normal samples $(p<0.001)$, as shown in Figure 6A. The expression levels of HSP90a were further compared in samples from different stages of tumor, but no significant difference was found, although the expression levels of HSP90a in samples from all stages were higher than these from normal samples, as shown in Figure 6B.

\section{DISCUSSION}

HSP90a, which has evolved for almost 3.5 billion years, is a member of the HSP90 family, a conserved and essential molecular chaperone, can be translocated to the cell surface and secreted into the extracellular space by cancer cells (Frydman, 2001; Eustace et al., 2004). The secreted HSP90a was associated with tumor development and prognosis, especially with cancer metastasis (Passarino et al., 2003; Eustace et al., 2004; Chang et al., 2009). Previously studies have showed that plasma HSP90a is an excellent biomarker for the diagnosis of lung cancer and liver cancer (Jhaveri et al., 2014; Shi et al., 2014; Fu et al., 2017). Meanwhile, plasma HSP90a levels were significantly higher than healthy controls in other cancers, but the diagnostic efficiency was insufficient, such as gastric cancer, breast cancer, nasopharyngeal carcinoma and colorectal cancer (Kasanga et al., 2018; Liu et al., 2019; Lin et al., 2020; Zhang et al., 2020). In current study, the plasma HSP90a levels was significantly higher in CRC patients than in healthy controls and consistent with previously reported results (Kasanga et al., 2018). Additionally, this current study finds that plasma HSP90a levels were associated with gender and disease progression as stage, lymphatic metastasis and distant metastasis in colorectal cancer patients. Plasma HSP90a levels were also observed in breast, liver and nasopharyngeal cancers to be closely correlated with disease stage and distant metastasis, but not with gender (Fu et al., 2017; Wei et al., 2020; Mao et al., 2021). Therefore, we speculate that the gender differences in HSP90a levels may be influenced by hormones. Multivariate statistical analysis also showed that the serum CEA, CA199 and plasma HSP90a are independent risk factors for distant metastasis,

TABLE 3 | Univariate and multivariate analysis for factors associated with metastasis in colorectal cancer.

\begin{tabular}{|c|c|c|c|c|}
\hline & Univariate analysis OR $(95 \% \mathrm{Cl})$ & $p$ value & Multivariate analysis OR $(95 \% \mathrm{Cl})$ & $p$ value \\
\hline Gender & $0.789(0.540,1.154)$ & 0.223 & - & - \\
\hline Age & $0.902(0.633,1.286)$ & 0.569 & - & - \\
\hline T cells & $0.988(0.970,1.005)$ & 0.174 & - & - \\
\hline Th cells & $0.994(0.973,1.015)$ & 0.564 & - & - \\
\hline Ts cells & $0.988(0.962,1.016)$ & 0.399 & - & - \\
\hline Ratio & $1.011(0.842,1.213)$ & 0.909 & - & - \\
\hline NK & $1.002(0.980,1.024)$ & 0.872 & - & - \\
\hline B cells & $0.999(0.965,1.034)$ & 0.940 & - & - \\
\hline CA153 & $1.024(0.999,1.050)$ & 0.063 & - & - \\
\hline AFP & $1.063(0.991,1.141)$ & 0.089 & - & - \\
\hline TK1 & $0.997(0.876,1.135)$ & 0.964 & - & - \\
\hline CA125 & $1.010(1.005,1.016)$ & $<0.001$ & $1.695(1.000,1.008)$ & 0.090 \\
\hline CEA & $1.008(1.005,1.011)$ & $<0.001$ & $3.647(1.003,1.008)$ & $<0.001$ \\
\hline CA199 & $1.003(1.002,1.004)$ & $<0.001$ & $5.454(1.001,1.003)$ & $<0.001$ \\
\hline HSP90a & $1.013(1.010,1.017)$ & $<0.001$ & 3.935 (1.004, 1.012) & $<0.001$ \\
\hline
\end{tabular}

The $\mathrm{p}$ values were shown in italics. 

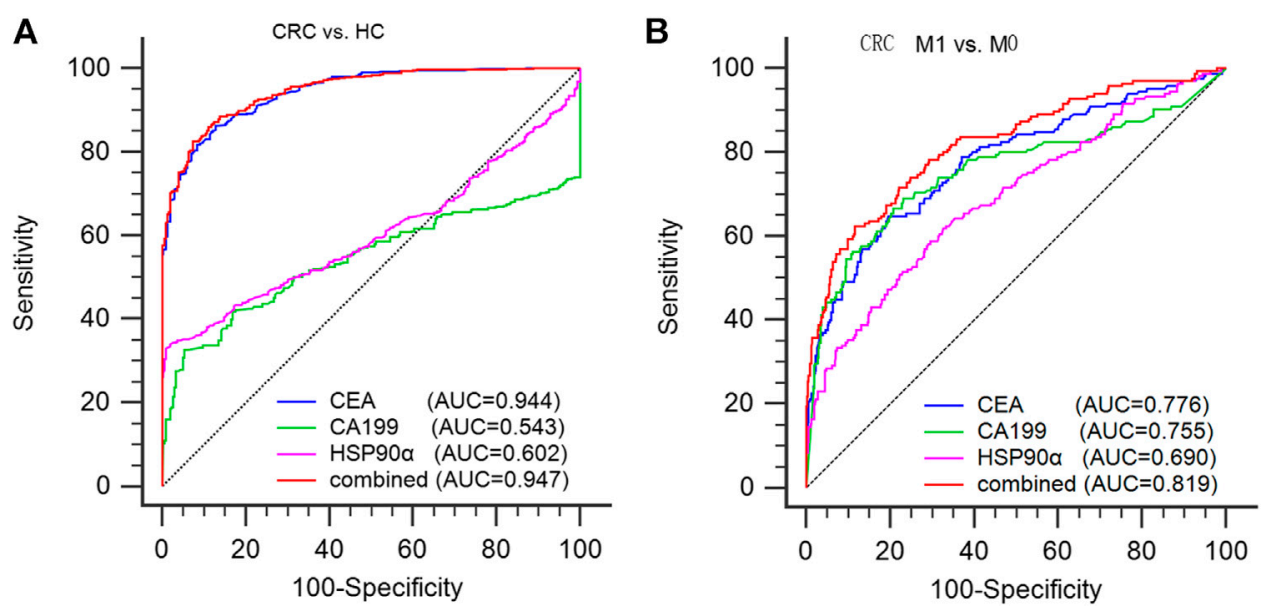

FIGURE 3 | diagnostic ability of serum CEA, CA199 and plasma HSP90a in colorectal cancer. (A) Using healthy donors as controls to evaluate the efficacy of CEA, CA199, HSP90 a and the panel in the diagnosis of colorectal cancer; (B) Using MO patients as controls to evaluate the efficacy of CEA, CA199, HSP90a and the panel for distinguishing the presence of distant metastasis from CRC patients. M0: no distant metastasis; M1: distant metastasis.

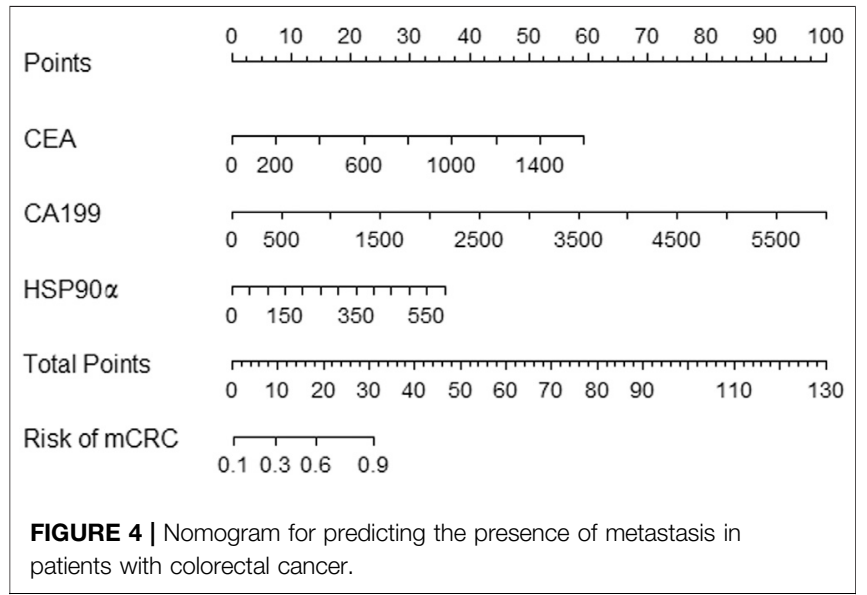

respectively. The Nomogram prediction of distant metastases in combination with CEA, CA199, and HSP90a can provide evidence for clinical treatment plans that may lead to better patient outcomes.

In correlation analysis, the common tumor makers CEA, AFP, CA125, CA153, CA199 showed no statistical correlation with immune cells. But it is worth noting that the plasma HSP90a levels was highly correlated with common tumor biomarkers as CEA, CA125, CA199 and immune cells as B lymphocyte that indicates that the expression of plasma HSP90a is closely related to the regulation of immune function. However, the mechanism of the association between plasma HSP90a and immune cells in the occurrence and progression of cancer is still unknown. It is well known that cancer is also an immune-related disease. At present, the detection of immune cells is one of the commonly used methods to monitor the body immunity in clinical practice. Previous studies have reported that NK cells percentage and B lymphocyte are associated with survival in
CRC patients (Berntsson et al., 2016; Tang et al., 2020). However, the plasma HSP90a levels were failed to find the significant prognosis value in colorectal cancer patients in this study. It's worth noting that the endpoint of most of the previous studies was overall survival (OS), while the endpoint of this study was PFS. Therefore, we speculated that although plasma HSP90a levels is not associated with PFS, it may be associated with OS in colorectal cancer patients and further research is needed to confirm this hypothesis.

Plasma HSP90a is overexpress and increases multimalignant phenotypes including chemoresistance to cisplatin as well as metastatic potentials in various types of cancers (Eustace et al., 2004; Chang et al., 2009; Zuehlke et al., 2015). Therefore, targeted treatment with HSP90a inhibitors offer interesting perspectives for the treatment of cancers. Previous studies have showed that cellular secretion of HSP90a from colorectal cancer cells was enhanced after serum starvation, and secreted HSP90a could be used to stimulate migration and invasion of other non-serum-starved cells, and the mechanism might be that secreted HSP90a acts through TCF12 expression to enhance CRC cell spreading (Chen et al., 2010; Chen et al., 2013). Currently, approximately 20 of these inhibitors have undergone clinical evaluation (Jhaveri et al., 2012; Jhaveri et al., 2014). A recent study reported that HSP90a inhibition sensitized immune-refractory tumor to adoptive $\mathrm{T}$ cell transfer as well as PD-1 blockade, and reinvigorated the immune cycle of tumor-reactive $\mathrm{T}$ cells (Song et al., 2020). From the results of this study, we know that the expression of plasma HSP90a is related to immune cells. Therefore, the use of HSP90a inhibitors alone or in combination with chemoradiotherapy or immunotherapy may be one of the treatment methods to improve the prognosis of metastatic colorectal cancer. At the same time, dynamic observation of changes of in plasma HSP90a levels may be one of the monitoring methods for the therapeutic efficacy of HSP90a inhibitors. 

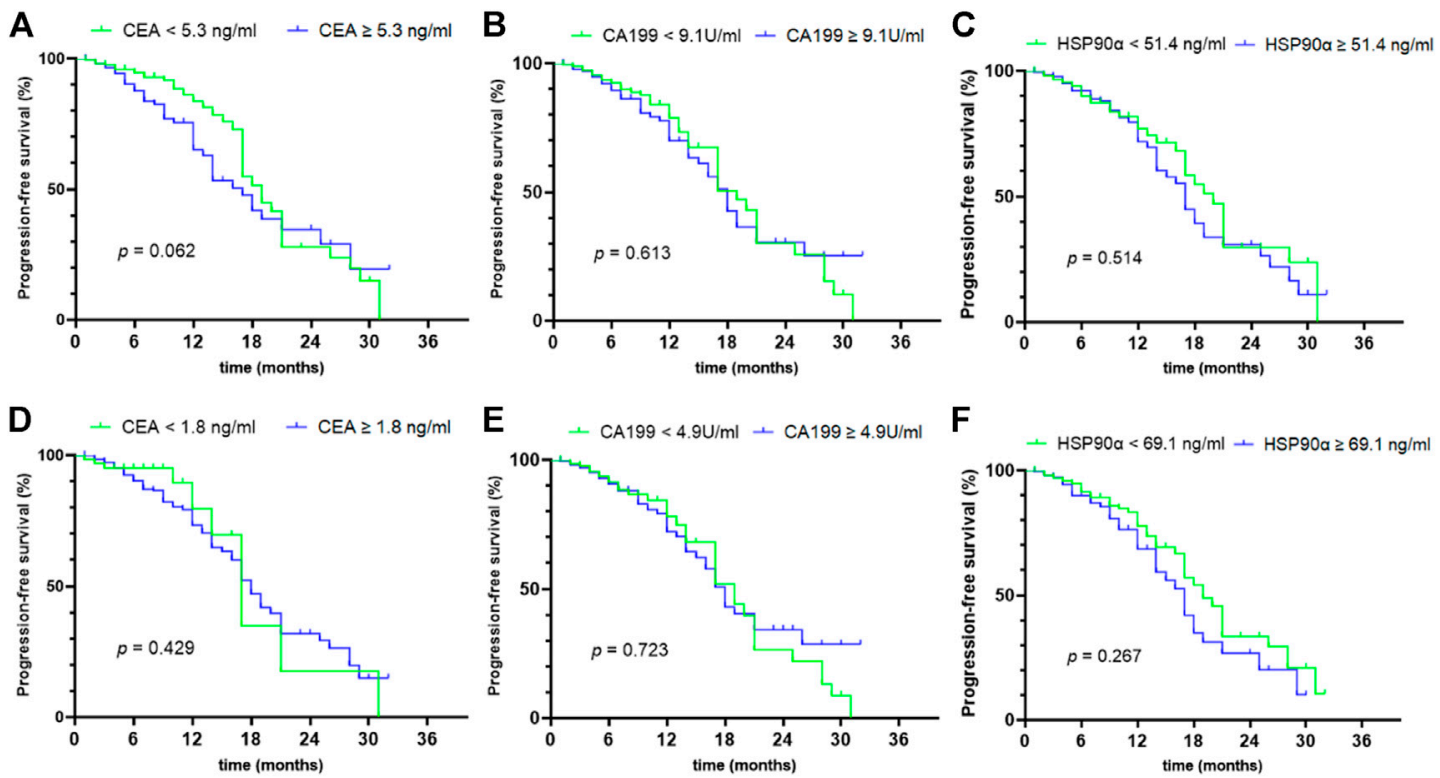

FIGURE 5 | prognostic value of initial concentrations of CEA, CA199 and HSP90a in colorectal cancer. (A-C) The prognostic values of initial concentrations of CEA, CA199 and HSP90a were analyzed by grouping based on median value; (D-F) The prognostic values of initial concentrations of CEA, CA199 and HSP90a were analyzed by grouping based on the cut-off value of ROC analysis in Figure $\mathbf{3 A}$.
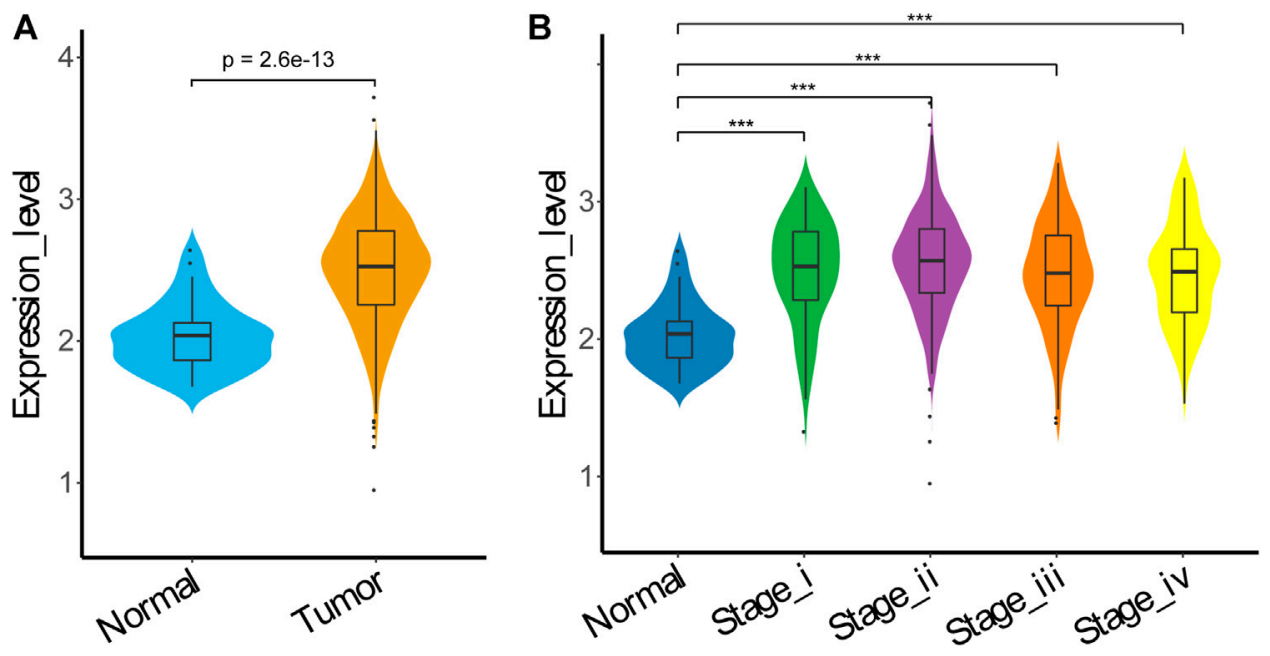

FIGURE 6 | HSP90 $a$ expression in TCGA COAD. (A) HSP90 $a$ expression in TCGA COAD samples from normal tissue $(n=40)$ or tumor tissue ( $n=458)$; (B) HSP90 a expression in TCGA COAD samples from normal tissue $(n=40)$ or different tumor stage tissue (stagel: $n=78$, stagell: $n=183$, stage III: $n=132$, stage IV: $n=65)$. Data were analyzed using Wilcoxon Rank Sum test.

\section{CONCLUSION}

The present study demonstrated that plasma Hsp90a was remarkable higher in colorectal cancer and correlated with common tumor biomarkers and immune cells. Plasma Hsp90a levels were associated with gender, stage, lymphatic metastasis and distant metastasis but a poor diagnosis performance in colorectal cancer. Meanwhile, the current study failed to show a prognostic value for plasma HSP90a in colorectal cancer.

\section{DATA AVAILABILITY STATEMENT}

The original contributions presented in the study are included in the article/supplementary material, further inquiries can be directed to the corresponding authors. 


\section{ETHICS STATEMENT}

The studies involving human participants were reviewed and approved by the Local Ethics Committee of Guangxi Medical University Cancer Hospital. The patients/participants provided their written informed consent to participate in this study.

\section{AUTHOR CONTRIBUTIONS}

WW, LZ, CZ, and HL conceived and designed the experiments; JZ and HL collected the patients' samples; WW, JZ, JL, SN, SL, and $\mathrm{CW}$ performed the experiments; WW, LC, and $\mathrm{YH}$ analyzed the data; WW wrote the paper; LZ, HL, and CZ revised the paper; All authors read and approved the final manuscript.

\section{REFERENCES}

Abdel-Rahman, O., and Cheung, W. Y. (2018). Integrating Systemic Therapies into the Multimodality Treatment of Resectable Colorectal Liver Metastases. Gastroent Res. Pract. 2018, 4326082. doi:10.1155/2018/4326082

Ahlenstiel, G., Hourigan, L. F., Brown, G., Zanati, S., Williams, S. J., Singh, R., et al. (2014). Actual Endoscopic versus Predicted Surgical Mortality for Treatment of Advanced Mucosal Neoplasia of the colon. Gastrointest. Endosc. 80 (4), 668-676. doi:10.1016/j.gie.2014.04.015

André, T., Boni, C., Navarro, M., Tabernero, J., Hickish, T., Topham, C., et al. (2009). Improved Overall Survival with Oxaliplatin, Fluorouracil, and Leucovorin as Adjuvant Treatment in Stage II or III Colon Cancer in the MOSAIC Trial. Jco 27 (19), 3109-3116. doi:10.1200/ jco.2008.20.6771

Baratti, D., Kusamura, S., Pietrantonio, F., Guaglio, M., Niger, M., and Deraco, M. (2016). Progress in Treatments for Colorectal Cancer Peritoneal Metastases during the Years 2010-2015. A Systematic Review. Crit. Rev. Oncol. Hematol. 100, 209-222. doi:10.1016/j.critrevonc.2016.01.017

Benson, A. B., 3rd, Bekaii-Saab, T., Chan, E., Chen, Y.-J., Choti, M. A., Cooper, H. S., et al. (2013). Metastatic Colon Cancer, Version 3.2013. J. Natl. Compr. Canc Netw. 11 (2), 141-152. doi:10.6004/jnccn.2013.0022

Berntsson, J., Nodin, B., Eberhard, J., Micke, P., and Jirström, K. (2016). Prognostic Impact of Tumour-Infiltrating B Cells and Plasma Cells in Colorectal Cancer. Int. J. Cancer 139 (5), 1129-1139. doi:10.1002/ijc.30138

Bray, F., Ferlay, J., Soerjomataram, I., Siegel, R. L., Torre, L. A., and Jemal, A. (2018). Global Cancer Statistics 2018: GLOBOCAN Estimates of Incidence and Mortality Worldwide for 36 Cancers in 185 Countries. CA: A Cancer J. Clin. 68 (6), 394-424. doi:10.3322/caac.21492

Chang, W., Ma, L., Lin, L., Gu, L., Liu, X., Cai, H., et al. (2009). Identification of Novel Hub Genes Associated with Liver Metastasis of Gastric Cancer. Int. J. Cancer 125 (12), 2844-2853. doi:10.1002/ijc.24699

Chen, J.-S., Hsu, Y.-M., Chen, C.-C., Chen, L.-L., Lee, C.-C., and Huang, T.-S. (2010). Secreted Heat Shock Protein 90a Induces Colorectal Cancer Cell Invasion through CD91/LRP-1 and NF-Kb-Mediated Integrin aV Expression. J. Biol. Chem. 285 (33), 25458-25466. doi:10.1074/jbc.m110.139345

Chen, W.-S., Chen, C.-C., Chen, L.-L., Lee, C.-C., and Huang, T.-S. (2013). Secreted Heat Shock Protein 90a (HSP90a) Induces Nuclear Factor-Kb-Mediated TCF12 Protein Expression to Down-Regulate E-Cadherin and to Enhance Colorectal Cancer Cell Migration and Invasion. J. Biol. Chem. 288 (13), 9001-9010. doi:10.1074/jbc.m112.437897

Cunningham, D., Lang, I., Marcuello, E., Lorusso, V., Ocvirk, J., Shin, D. B., et al. (2013). Bevacizumab Plus Capecitabine versus Capecitabine Alone in Elderly Patients with Previously Untreated Metastatic Colorectal Cancer (AVEX): an Open-Label, Randomised Phase 3 Trial. Lancet Oncol. 14 (11), 1077-1085. doi:10.1016/s1470-2045(13)70154-2

Eisenhauer, E. A., Therasse, P., Bogaerts, J., Schwartz, L. H., Sargent, D., Ford, R., et al. (2009). New Response Evaluation Criteria in Solid Tumours: Revised

\section{FUNDING}

This work was supported by the National Science Foundation of Guangxi Province of China (Grant No. 2017GXNSFAA198155); Guangxi Scientific Research and Technical Planning Project (Gui ke AB19110018); and the Scientific Research and Technical Development Project of Qingxiu District, Nanning City, Guangxi Province (Nos. 2017034 and 2016051).

\section{ACKNOWLEDGMENTS}

The authors would like to thank Dev Sooranna, Imperial College London, for editing the manuscript.

RECIST Guideline (Version 1.1). Eur. J. Cancer 45 (2), 228-247. doi:10.1016/ j.ejca.2008.10.026

Eustace, B. K., Sakurai, T., Stewart, J. K., Yimlamai, D., Unger, C., Zehetmeier, C., et al. (2004). Functional Proteomic Screens Reveal an Essential Extracellular Role for Hsp90a in Cancer Cell Invasiveness. Nat. Cel Biol 6 (6), 507-514. doi:10.1038/ncb1131

Frydman, J. (2001). Folding of Newly Translated Proteins In Vivo: The Role of Molecular Chaperones. Annu. Rev. Biochem. 70, 603-647. doi:10.1146/ annurev.biochem.70.1.603

Fu, Y., Xu, X., Huang, D., Cui, D., Liu, L., Liu, J., et al. (2017). Plasma Heat Shock Protein 90alpha as a Biomarker for the Diagnosis of Liver Cancer: An Official, Large-Scale, and Multicenter Clinical Trial. Ebiomedicine 24, 56-63. doi:10.1016/j.ebiom.2017.09.007

Haller, D. G., Tabernero, J., Maroun, J., de Braud, F., Price, T., Van Cutsem, E., et al. (2011). Capecitabine Plus Oxaliplatin Compared with Fluorouracil and Folinic Acid as Adjuvant Therapy for Stage III Colon Cancer. Jco 29 (11), 1465-1471. doi:10.1200/jco.2010.33.6297

Hurwitz, H., Fehrenbacher, L., Novotny, W., Cartwright, T., Hainsworth, J., Heim, W., et al. (2004). Bevacizumab Plus Irinotecan, Fluorouracil, and Leucovorin for Metastatic Colorectal Cancer. N. Engl. J. Med. 350 (23), 2335-2342. doi:10.1056/nejmoa032691

Jhaveri, K., Taldone, T., Modi, S., and Chiosis, G. (2012). Advances in the Clinical Development of Heat Shock Protein 90 (Hsp90) Inhibitors in Cancers. Biochim. Biophys. Acta (Bba) - Mol. Cel Res. 1823 (3), 742-755. doi:10.1016/ j.bbamcr.2011.10.008

Jhaveri, K., Ochiana, S. O., Dunphy, M. P., Gerecitano, J. F., Corben, A. D., Peter, R. I., et al. (2014). Heat Shock Protein 90 Inhibitors in the Treatment of Cancer: Current Status and Future Directions. Expert Opin. Investig. Drugs 23 (5), 611-628. doi:10.1517/13543784.2014.902442

Kasanga, M., Liu, L., Xue, L., and Song, X. (2018). Serum Heat Shock Protein 90a Have an Advantage in Diagnosis of Colorectal Cancer at Early Stage. Biomarkers Med. 12 (8), 881-890. doi:10.2217/bmm-2018-0155

Leonard, G. D., Brenner, B., and Kemeny, N. E. (2005). Neoadjuvant Chemotherapy before Liver Resection for Patients with Unresectable Liver Metastases from Colorectal Carcinoma. Jco 23 (9), 2038-2048. doi:10.1200/ jco.2005.00.349

Lin, T., Qiu, Y. Q., Peng, W. Y., and Peng, L. S. (2020). Heat Shock Protein 90 Family Isoforms as Prognostic Biomarkers and Their Correlations with Immune Infiltration in Breast Cancer. Biomed. Res. Int., 2020, 2148253. doi:10.1155/2020/2148253

Liu, F. X., Wang, L. L., Yi, S. J., Liu, Q. H., Xu, X. X., and Su, M. (2019). Clinical and Biological Significances of Heat Shock Protein 90 (Hsp90) in Human Nasopharyngeal Carcinoma Cells and Anti-cancer Effects of Hsp90 Inhibitor. Biomed. Pharmacother. 120, 109533 . doi:10.1016/ j.biopha.2019.109533

Ma, B., Gao, P., Wang, H., Xu, Q., Song, Y., Huang, X., et al. (2017). What Has Preoperative Radio(chemo)therapy Brought to Localized Rectal Cancer Patients in Terms of Perioperative and Long-Term Outcomes over the Past 
Decades? A Systematic Review and Meta-Analysis Based on 41,121 Patients. Int. J. Cancer 141 (5), 1052-1065. doi:10.1002/ijc.30805

Manfredi, S., Lepage, C. m., Hatem, C., Coatmeur, O., Faivre, J., and Bouvier, A.-M. (2006). Epidemiology and Management of Liver Metastases from Colorectal Cancer. Ann. Surg. 244 (2), 254-259. doi:10.1097/01.sla.0000217629.94941.cf

Mao, M., Wang, X., Sheng, H., Li, H., Liu, W., Han, R., et al. (2021). Heat Shock Protein 90a Provides an Effective and Novel Diagnosis Strategy for Nasopharyngeal Carcinoma. Adv. Ther. 38 (1), 413-422. doi:10.1007/ s12325-020-01518-4

Mohammad, W., and Balaa, F. (2009). Surgical Management of Colorectal Liver Metastases. Clin. colon rectal Surg. 22 (4), 225-232. doi:10.1055/s-00291242462

Morris, E., and Treasure, T. (2018). Surgical Management and Outcomes of Colorectal Cancer Liver Metastases. Cancer Epidemiol. 52, 160-161. doi:10.1016/j.canep.2017.12.010

Passarino, G., Cavalleri1, G. L., Stecconi, R., Franceschi, C., Altomare, K., Dato, S., et al. (2003). Molecular Variation of Human HSP90 $\alpha$ and HSP90 $\beta$ Genes in Caucasians. Hum. Mutat. 21 (5), 554-555. doi:10.1002/humu.9141

Rojas Llimpe, F. L., Di Fabio, F., Ercolani, G., Giampalma, E., Cappelli, A., Serra, C., et al. (2014). Imaging in Resectable Colorectal Liver Metastasis Patients with or without Preoperative Chemotherapy: Results of the PROMETEO-01 Study. Br. J. Cancer 111 (4), 667-673. doi:10.1038/bjc.2014.351

Saltz, L. B., Clarke, S., Díaz-Rubio, E., Scheithauer, W., Figer, A., Wong, R., et al. (2008). Combination with Oxaliplatin-Based Chemotherapy as First-Line Therapy in Metastatic Colorectal Cancer: A Randomized Phase III Study. J. Clin. Oncol. 26 (12), 2013-2019. doi:10.1200/JCO.2007.14.9930

Shi, Y., Liu, X., Lou, J., Han, X., Zhang, L., Wang, Q., et al. (2014). Plasma Levels of Heat Shock Protein 90 Alpha Associated with Lung Cancer Development and Treatment Responses. Clin. Cancer Res. 20 (23), 6016-6022. doi:10.1158/10780432.ccr-14-0174

Song, K. H., Oh, S. J., Kim, S., Cho, H., Lee, H. J., Song, J. S., et al. (2020). HSP90A Inhibition Promotes Anti-tumor Immunity by Reversing Multi-Modal Resistance and Stem-like Property of Immune-Refractory Tumors. Nat. Commun. 11 (1), 562. doi:10.1038/s41467-019-14259-y

Tang, Y. P., Xie, M. Z., Li, K. Z., Li, J. L., Cai, Z. M., and Hu, B. L. (2020). Prognostic Value of Peripheral Blood Natural Killer Cells in Colorectal Cancer. Bmc Gastroenterol. 20 (1), 31. doi:10.1186/s12876-020-1177-8
Tsutsumi, S., Scroggins, B., Koga, F., Lee, M.-J., Trepel, J., Felts, S., et al. (2008). A Small Molecule Cell-Impermeant Hsp90 Antagonist Inhibits Tumor Cell Motility and Invasion. Oncogene 27 (17), 2478-2487. doi:10.1038/ sj.onc. 1210897

Van Cutsem, E., Köhne, C.-H., Hitre, E., Zaluski, J., Chang Chien, C.-R., Makhson, A., et al. (2009). Cetuximab and Chemotherapy as Initial Treatment for Metastatic Colorectal Cancer. N. Engl. J. Med. 360 (14), 1408-1417. doi:10.1056/nejmoa0805019

van Kessel, C. S., Buckens, C. F. M., van den Bosch, M. A. A. J., van Leeuwen, M. S., van Hillegersberg, R., and Verkooijen, H. M. (2012). Preoperative Imaging of Colorectal Liver Metastases after Neoadjuvant Chemotherapy: a Meta-Analysis. Ann. Surg. Oncol. 19 (9), 2805-2813. doi:10.1245/s10434-012-2300-z

Wei, W., Liu, M. S., Ning, S. F., Wei, J., Zhong, J. H., Li, J. L., et al. (2020). Diagnostic Value of Plasma HSP90 Alpha Levels for Detection of Hepatocellular Carcinoma. Bmc Cancer 20 (1). doi:10.1186/s12885-0196489-0

Zhang, Y., Ni, L., Li, Q., Li, M., Zhu, J., Zhang, F., et al. (2020). Diagnostic, Clinicopathologic, Therapeutic and Prognostic Value of Plasma Heat Shock Protein 90 Levels in Patients with Advanced Gastrointestinal Carcinoma. J. Cancer 11 (20), 5890-5899. doi:10.7150/jca.46343

Zuehlke, A. D., Beebe, K., Neckers, L., and Prince, T. (2015). Regulation and Function of the Human HSP90AA1 Gene. Gene 570 (1), 8-16. doi:10.1016/ j.gene.2015.06.018

Conflict of Interest: The authors declare that the research was conducted in the absence of any commercial or financial relationships that could be construed as a potential conflict of interest.

The handling editor declared a past collaboration with one of the authors CZ.

Copyright $\odot 2021$ Wei, Zhou, Chen, Liu, Zhang, Li, Ning, Li, Wang, Huang, Zou and Zhang. This is an open-access article distributed under the terms of the Creative Commons Attribution License (CC BY). The use, distribution or reproduction in other forums is permitted, provided the original author(s) and the copyright owner(s) are credited and that the original publication in this journal is cited, in accordance with accepted academic practice. No use, distribution or reproduction is permitted which does not comply with these terms. 